\title{
Long-term survival rates of patients with stage IIIB and IV non-small cell lung cancer treated with cisplatin plus vinorelbine or gemcitabine
}

\author{
SEVKET OZKAYA $^{1}$, SERHAT FINDIK ${ }^{2}$, ADEM DIRICAN $^{3}$ and ATILLA GÜVEN ATICI ${ }^{2}$ \\ ${ }^{1}$ Department of Pulmonary Medicine, Faculty of Medicine, Rize University, Rize; ${ }^{2}$ Department of Pulmonary Medicine, \\ Faculty of Medicine, Ondokuz Mayis University, Samsun; ${ }^{3}$ Department of Pulmonary Medicine, \\ Samsun Medical Park Hospital, Samsun, Turkey
}

Received March 6, 2012; Accepted May 3, 2012

DOI: $10.3892 /$ etm.2012.714

\begin{abstract}
Limited data exist concerning the long-term ( $\geq 5$ year) survival rates of patients with stage IIIB and IV nonsmall cell lung carcinoma (NSCLC) receiving chemotherapy. We aimed to determine the long-term results of cisplatin plus third-generation (vinorelbine or gemcitabine) cytotoxic chemotherapy in patients with locally advanced and advanced NSCLC. The study included 141 patients, and all patients were followed up from the time of diagnosis until death. The median age of the patients was $59.1 \pm 9.9$ years. The male-to-female ratio was $124 / 17 ; 62.4 \%$ of the patients had stage IIIB and $37.6 \%$ had stage IV NSCLC. Squamous cell carcinoma, adenocarcinoma and undifferentiated NSCLC subtypes accounted for 69.5, 17.7 and $12.7 \%$ of the cases, respectively. The overall response rate was $32.6 \%$ and the median survival time was 12.3 months (95\% CI, 10.2-14.5). The median survival times for stages IIIB and IV were $12.6 \pm 1.4$ and $11.9 \pm 1.7$ months, respectively. The 1-, 2-, 3- and 5-year survival rates were 33, 7.5, 4.3 and $2.8 \%$, respectively. In conclusion, cisplatin-based new-generation cytotoxic agents for combined modality therapy offer an increased hope of long-term survival for patients with locally advanced and advanced NSCLC.
\end{abstract}

\section{Introduction}

Lung cancer is the leading cause of cancer-related death in the world, and non-small cell lung cancer (NSCLC) accounts for $80-85 \%$ of lung cancer cases. Patients with early-stage NSCLC have relatively high long-term survival rates after surgical resection, but a substantial majority of patients, $\sim 80 \%$, present in advanced or metastatic stages. Over the past decade,

Correspondence to: Dr Sevket Ozkaya, Department of Pulmonary Medicine, Faculty of Medicine, Rize University, Rize, Turkey E-mail: ozkayasevket@yahoo.com

Key words: non-small cell lung cancer, long-term survival, cisplatin, vinorelbine, gemcitabine third-generation agents such as vinorelbine, taxanes and gemcitabine have been introduced for the treatment of NSCLC (1). Combination of one or more of these agents with a platinum compound has resulted in high response rates and prolonged overall survival. Today, doublet chemotherapies consisting of platinum plus one of the third-generation agents have become the current standard regimen, the first line of chemotherapy (2). Many studies have examined short-term survival rates in patients receiving such treatment, yet current evidence regarding long-term survival in advanced-stage NSCLC, particularly in stages IIIB and IV, is limited. This study aimed to determine the long-term results of cisplatin plus third-generation (vinorelbine or gemcitabine) cytotoxic chemotherapy in patients with locally advanced and advanced NSCLC.

\section{Materials and methods}

Patients. The patients with stage IIIB and IV NSCLC were evaluated at the Department of Pulmonary Medicine, Ondokuz Mayis University's Faculty of Medicine, between January 2001 and September 2004. The patient data, which included demographic, clinical, radiological, disease characteristics and therapy regimens were retrospectively obtained from the files of the patients in departmental archives. We followed up the patients from the time of diagnosis until death, and all of the patients had succumbed to causes related to lung cancer. A total of 196 patients were enrolled in the study, however, $55(28 \%)$ were excluded due to factors rendering them inappropriate for the study and thus 141 patients were included. Approval from the patients and the institution was obtained in order to use their records for our study.

Eligibility criteria. Performance status was classified in accordance with the criteria of the European Cooperative Oncology Group (ECOG). Staging was conducted by evaluation of imaging methods, chest X-ray, thoracic computed tomography, abdominal computed tomography, abdominal ultrasonography (USG), cranial computed tomography and bone scintigraphy. The criteria for eligibility included pathologically confirmed NSCLC, radiologically measurable lesions, an age of at least 18 years, adequate hematological function (as indicated by a 
white cell count of at least $4,000 / \mathrm{ml}^{3}$ and a platelet count of at least $100,000 / \mathrm{ml}^{3}$ ), hepatic function (as indicated by a bilirubin level that did not exceed $1.5 \mathrm{mg} / \mathrm{dl}$, and by AST and ALT levels being $<3$ times the normal values), renal function (as indicated by a creatinine level that did not exceed $1.5 \mathrm{mg} / \mathrm{dl}$ ), and ECOG performance status (PS) $\leq 2$. Criteria for exclusion from the study were as follows: insufficient hematological, renal or hepatic functions; unstable brain metastasis; history of prior chemotherapy and/or radiotherapy; presence of uncontrolled infections; presence of an additional malignancy; presence of a systemic disease contradicting administration of chemotherapy; pregnancy; ECOG PS >3; and unfitness for follow-up due to psychological, familial, sociological or geographical reasons.

Treatment plan. The main treatment regimen consisted of chemotherapy for patients in stage IV of the disease, and sequential chemoradiotherapy was used for patients in stage IIIB. Vinorelbine at a dose of $30 \mathrm{mg} / \mathrm{m}^{2}$ or gemcitabine at a dose of $1,250 \mathrm{mg} / \mathrm{m}^{2}$ on Days 1 and 8 and cisplatin at a dose of $80 \mathrm{mg} / \mathrm{m}^{2}$ on Day 1 were administered on a threeweek cycle. The cycle was repeated every three weeks. At least two cycles were administered to the patients who were considered assessable for response. Patients who responded to the treatment and did not show signs of toxicity or progression received four to six cycles. Dosage was adjusted according to hematological, neurological, renal and hepatic functions. Dosage was decreased by $25 \%$ for patients who were classified as Grade III or Grade IV in accordance with WHO toxicity criteria. Curative radiotherapy was administered to all patients in stage IIIB of the disease who responded to chemotherapy after three cycles of chemotherapy regimens. A final one to three cycles were administered between three weeks and one month after administration of radiotherapy. Standard ECOG response criteria were used. The response was evaluated by thorax CT scan after two cycles of chemotherapy and at the end of the treatment. Briefly, a complete response was defined as the absence of disease at all known sites for at least four weeks. A partial response was defined as a $50 \%$ reduction in the sum of the perpendicular diameters of all measurable lesions, lasting at least four weeks. Progressive disease was defined as either a $25 \%$ increase in the area of any one lesion over the prior measurement or the development of one or more new lesions. Survival was calculated from the date of diagnosis until the date of death.

Statistical analysis. Data were evaluated with SPSS 13.0 (SPSS, Inc., Chicago, IL, USA). Survival of the patients was calculated from the date of diagnosis to the date of death. Response rates were calculated for patients with complete or partial responses. Median age, smoking habits, performance status, response rates and toxicity results of the groups were compared with the Mann-Whitney U and Pearson Chi-square tests. The survival rates were calculated by the Kaplan-Meier method.

\section{Results}

The median age was $59.1 \pm 9.9$ years and the male-to-female ratio was 124/17. Most of the patients had smoked $>21$ packs/ year $(81.6 \%)$. In the distribution of ECOG PS, $46.1 \%$ of patients
Table I. Baseline characteristics of the patients.

\begin{tabular}{|c|c|}
\hline Characteristics & $\mathrm{n}=141$ \\
\hline Age (median \pm SD) & $59.1 \pm 9.9$ \\
\hline \multicolumn{2}{|l|}{ Gender $(\%)$} \\
\hline Male & 87.9 \\
\hline Female & 12.1 \\
\hline \multicolumn{2}{|l|}{ Smoking status (\%) } \\
\hline Nonsmoker & 5.0 \\
\hline$<10$ packs/year & 5.0 \\
\hline 10-20 packs/year & 8.5 \\
\hline 21-30 packs/year & 20.6 \\
\hline$>30$ packs/year & 61.0 \\
\hline \multicolumn{2}{|c|}{ ECOG performance status (\%) } \\
\hline $0-1$ & 46.1 \\
\hline 2 & 43.3 \\
\hline $3-4$ & 10.6 \\
\hline \multicolumn{2}{|l|}{ Disease stage $(\%)$} \\
\hline IIIB & 62.4 \\
\hline IV & 37.6 \\
\hline \multicolumn{2}{|l|}{ Histological type (\%) } \\
\hline Squamous cell & 69.5 \\
\hline Adenocarcinoma & 17.7 \\
\hline Undifferentiated NSCLC & 12.7 \\
\hline \multicolumn{2}{|l|}{ Metastatic sites (\%) } \\
\hline Bone & 15.0 \\
\hline Brain & 10.5 \\
\hline Liver & 6.0 \\
\hline Adrenal & 4.5 \\
\hline
\end{tabular}

were in ECOG 0-1, $43.3 \%$ of patients in ECOG 2 and $10.6 \%$ of patients in ECOG 3-4. It was observed that $62.4 \%$ of the patients had stage IIIB disease and $37.6 \%$ of patients had stage IV disease. Furthermore, $69.6 \%$ of patients had squamous cell carcinoma, $17.7 \%$ of patients had adenocarcinoma and $12.7 \%$ of patients were classified as having undifferentiated NSCLC. The metastatic sites were bone (15\%), brain (10.5\%), liver $(6 \%)$ and adrenal gland $(4.5 \%)$ in the metastatic patients (Table I). The median number of chemotherapy cycles was 3.7 for cisplatin plus third-generation (vinorelbine or gemcitabine) agents. The overall response rate was $32.6 \%$. Respectively, 32.9 and $18 \%$ of patients received curative and palliative radiotherapy, and $13.5 \%$ of patients received second-line chemotherapy. The median survival time was 12.3 months (95\% CI, 10.2-14.5). The median survival times for stages IIIB and IV were 12.6 \pm 1.4 and 11.9 \pm 1.7 months, respectively. The 1-, 2-, 3- and 5-year survival rates were $33,7.5,4.3$ and $2.8 \%$, respectively (Table II). All patients were evaluated for toxicity. The major hematological toxicities encountered in this study were neutropenia, febrile neutropenia, thrombocytopenia and anemia. Percentages of grades 1-2 and 3-4 toxicity for anemia were 43.9 and $8.1 \%$, for neutropenia 40.2 and $9.6 \%$, for thrombocytopenia 8.9 and $5.1 \%$ and for nausea and vomiting 61.9 and $3.6 \%$, respectively. The rate of febrile neutropenia was $4.9 \%$ (Table III). No serious 
Table II. Outcomes of the treatments.

\begin{tabular}{lc}
\hline Variables & $\mathrm{n}=141$ \\
\hline Response (\%) & \\
Complete response & 6.8 \\
Partial response & 26.1 \\
Stable disease & 40.5 \\
Progressive disease & 26.6 \\
Overall response rate (\%) & 32.6 \\
Radiotherapy (\%) & \\
Curative & 32.9 \\
Palliative & 18.0 \\
Second-line chemotherapy (\%) & 13.5 \\
Overall survival [months (95\% CI)] & $(10.2-14.5)$ \\
Stage IIIB median survival (months) & $12.6 \pm 1.4$ \\
Stage IV median survival (months) & $11.9 \pm 1.7$ \\
Survival (\%) & \\
1-year survival & 33.0 \\
2-year survival & 7.5 \\
3-year survival & 4.3 \\
5-year survival & 2.8 \\
\hline
\end{tabular}

Table III. Toxic effects.

\begin{tabular}{lr}
\hline Type of toxicity & $\mathrm{n}=141$ \\
\hline Anemia (\%) & \\
Grade 1-2 & 43.9 \\
Grade 3-4 & 8.1 \\
Neutropenia (\%) & 40.2 \\
Grade 1-2 & 9.6 \\
Grade 3-4 & 4.9 \\
Febrile neutropenia (\%) & 8.9 \\
Thrombocytopenia (\%) & 5.1 \\
Grade 1-2 & \\
Grade 3-4 & 61.9 \\
Nausea and vomiting (\%) & 3.6 \\
Grade 1-2 & \\
Grade 3-4 &
\end{tabular}

hemorrhagic events were noted with either regimen. KaplanMeier survival curves of all patients, stage III and IV are shown in Figs. 1 and 2.

\section{Discussion}

According to reported studies, the long-term survival rate of patients with locally advanced and advanced NSCLC, varies by disease characteristics but is generally low, with 5-year

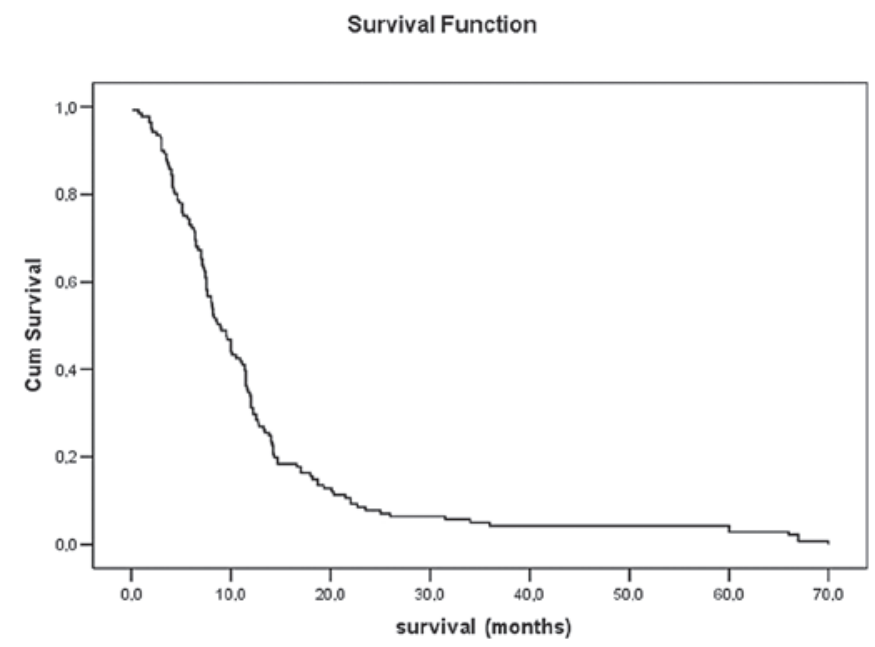

Figure 1. Kaplan-Meier survival curve of all patients.

Survival Functions

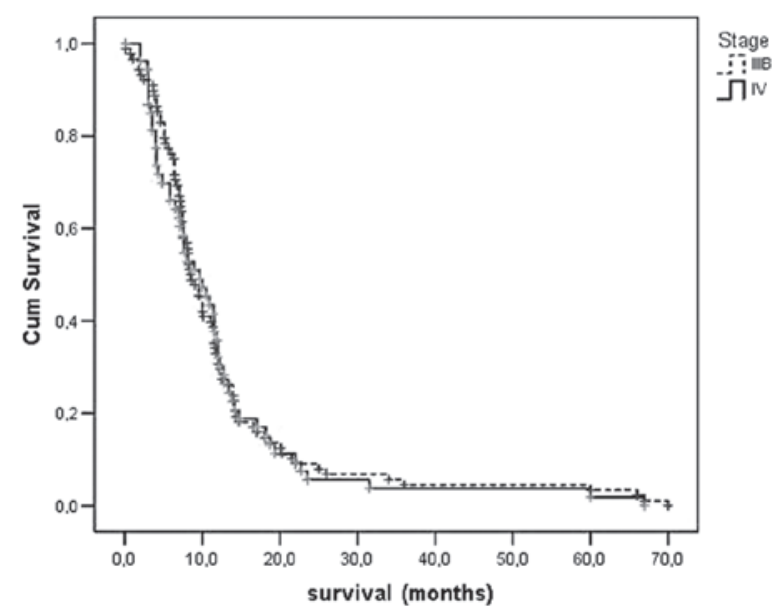

Figure 2. Kaplan-Meier survival curves of patients with stage III and IV.

survival rates for all stages ranging from 9 to $15 \%$. The reported survival rates in stages IIIA (14.1\%), IIIB (4.6\%) and IV $(4.2 \%)$ are closely comparable to those reported from other centers for IIIA (8-11\%), IIIB (1-5\%) and IV (1-5\%) (3-5).

Hagerty et al (6) emphasized the importance of patient preferences in the case of metastatic disease from a variety of cancers, with patients more frequently wanting to know the longest survival time with treatment rather than the 5-year survival rate. However, data are sparse concerning the 5-year survival rates and 5-year survival advantages of patients with stage IIIB and IV NSCLC who receive chemotherapy or chemoradiotherapy.

Okamoto et al (7) reported that $7.7 \%$ of 222 metastatic NSCLC patients survived for $>2$ years. Satoh et al (8) reported that 19.4 and $13.9 \%$ of advanced NSCLC patients survived for $>2$ or 3 years with cisplatin-based chemotherapy, respectively. In this study, all patients received platinum-based chemotherapy as a first-line chemotherapy, and the response rate was found to be $42.8 \%$. Kaira et al (9) reported a $20 \%$ response rate with first-line chemotherapy and $8 \%$ of patients survived 
for $>5$ years. These findings are similar to the findings of our study. Wang et al (5) evaluated 56 patients with stage III and IV NSCLC who had survived for 5 years or longer. Only one (1.7\%) patient with stage IV NSCLC survived for 5 years treated with chemotherapy alone.

There is a wide variety in the toxicity rates reported in previous studies, with grade 3-4 anemia in 7-24\% and $20-30 \%$ of the patients receiving cisplatin and vinorelbine and the patients receiving cisplatin and gemcitabine, respectively; grade 3-4 neutropenia in 5.4-38.5\% and 13.8-81\%; grade 3-4 thrombocytopenia in 2.5-20\% and 2.5-6\%; and grade 3-4 nausea and vomiting in $0-58 \%$ and $3.2-39 \%$, respectively (10-13). Our toxicity results were consistent with those of previous studies.

In conclusion, the effects of chemotherapy in advancedstage NSCLC patients have been controversial since the 1990s. However, the cisplatin-based new-generation cytotoxic agents for combined modality therapy offer increased hope of longterm survival of patients with locally advanced and advanced NSCLC. There is a continued need to follow up the outcomes of such patients over long periods of time.

\section{References}

1. Molina JR, Adjei AA and Jett JR: Advances in chemotherapy of non-small cell lung cancer. Chest 130: 1211-1219, 2006.

2. Pfister DG, Johnson DH, Azzoli CG, Sause W, Smith TJ, Baker S Jr, et al: American Society of Clinical Oncology treatment of unresectable non-small-cell lung cancer guideline: update 2003. J Clin Oncol 22: 330-353, 2004.

3. De Cos Escuin JS, Vecente CD, Penafiel JC, Miranda JAR, Gonzalez MAS and Jimenez JFM: Overall long-term survival in lung cancer analyzed in 610 unselected patients. Arch Bronconeumol 40: 268-274, 2004.
4. Fry WA, Phillips JL and Menck HR: Ten-year survey of lung cancer treatment and survival in hospitals in the United States: a national cancer data base report. Cancer 86: 1867-1876, 1999.

5. Wang T, Nelson RA, Bogardus A and Granis FW Jr: Five-year lung cancer survival: which advanced stage nonsmall cell lung cancer patients attain long-term survival? Cancer 116: 1518-1525, 2010.

6. Hagerty RG, Butow PN, Ellis PA, et al: Cancer patient preferences for communication of prognosis in the metastatic setting. J Clin Oncol 22: 1721-1730, 2004.

7. Okamoto T, Maruyama R, Shoji F, Asoh H, Ikeda J, Miyamoto T, et al: Long-term survivors in stage IV non-small cell lung cancer. Lung Cancer 47: 85-91, 2005.

8. Satoh H, Ishikawa H, Ohara G, Kagohashi K, Kurishima K, Ohtsuka $\mathrm{M}$ and Hizawa N: Long-term survivors after chemotherapy in advanced non-small cell lung cancer. Anticancer Res 27: 4457-4460, 2007.

9. Kaira K, Takahashi T, Murakami H, Tsuya A, Nakamura Y, Naito T, Endo M and Yamamoto N: Long-term survivors of more than 5 years in advanced non-small cell lung cancer. Lung Cancer 67: 120-123, 2010.

10. Southern Italy Cooperative Oncology Group (SICOG): Gemcitabine plus cisplatin combinations in advanced non-small cell lung cancer. Anticancer Drugs 11: S23-S27, 2000.

11. Huisman C, Giaccone G, van Groeningen CJ, Sutedja G, Postmus PE and Smit EF: Combination of gemcitabine and cisplatin for advanced non-small cell lung cancer: a phase II study with emphasis on scheduling. Lung Cancer 33: 267-275, 2001.

12. Crino L, Scagliotti GV, Ricci S, De Marinis F, Rinaldi M, Gridelli C, et al: Gemcitabine and cisplatin versus mitomycin, ifosfamide, and cisplatin in advanced non-small-cell lung cancer: a randomized phase III study of the Italian Lung Cancer Project. J Clin Oncol 17: 3522-3530, 1999.

13. Cardenal F, Lopez-Cabrerizo M, Anton A, Alberola V, Massuti B, Carrato A, et al: Randomized phase III study of gemcitabine-cisplatin versus etoposide-cisplatin in the treatment of locally advanced or metastatic non-small-cell lung cancer. J Clin Oncol 17: 12-18, 1999. 